\title{
CLASES Y FUNCIONES EN EL ÁMBITO MORFOLÓGICO*
}

\section{CATEGORIES AND FUNCTIONS IN MORPHOLOGY}

\author{
Luis GonZÁlez García \\ Universidade da Coruña \\ Grupo Hispania \\ luis.gonzalezg@udc.es
}

Enviado: 07/03/2018

Aceptado: 07/09/2018

\begin{abstract}
Resumen
La distinción entre clases de unidades y las funciones que estas desempeñan en el nivel sintáctico ha sido objeto de estudio por parte de las distintas escuelas lingüísticas (funcionalistas o formalistas), si bien desde postulados divergentes. Sin embargo, esta esencial dicotomía apenas ha recibido atención en el componente morfológico, siendo lo usual que se entremezclen los dos puntos de vista. En este trabajo mostramos que esa distinción también debe ser trasladada al campo de la morfología, al tiempo que ofrecemos algunas muestras de cómo su utilización permite tratar de manera satisfactoria algunas cuestiones objeto de debate en el análisis morfológico del español (por ejemplo, los interfijos o la oposición lexema/raíz).

PALABRAS CLAVE: unidades morfológicas, clases de morfemas, análisis morfológico, morfología del español, formación de palabras.
\end{abstract}

\begin{abstract}
The distinction between form classes and the functions they play in the syntactic level has been studied by different grammatical theories (functionalist or formalist), although from divergent postulates. However, this essential dichotomy has received little attention in morphology, so it is usual that the two approaches get mixed up. Therefore, in this paper I will show that this distinction must also be transferred to the field of morphology. At the same time, I will present some samples of how the use of this twofold perspective allows us to treat some issues under discussion in the morphological analysis (e.g., interfixes or the distinction lexeme/root) in a satisfactory way.

KEY WORDS: Morphological units, classes of morphemes, morphological analysis, Spanish morphology, word formation.
\end{abstract}

* Una versión previa, reducida, de este trabajo fue presentada en el VIII Congreso Internacional de Lingüística Hispánica (Leipzig, septiembre de 2016). Este trabajo se completó en el marco de la ayuda para la consolidación y la estructuración de unidades de investigación competitivas del Sistema universitario de Galicia concedida al Grupo Hispania de la Universidad de A Coruña, referencia GPC2015/028 (2015-2017), de la Consellería de Cultura, Educación e Ordenación Universitaria (Xunta de Galicia).

Para citar este artículo / To cite this article: González García, Luis (2018). Clases y funciones en el ámbito morfológico. ELUA, 32: 179-193. doi: 10.14198/ELUA2018.32.8

Enlace / Link: http://dx.doi.org/10.14198/ELUA2018.32.8 


\section{INTRODUCCIÓN}

La distinción entre clases y funciones es un principio básico, asentado desde los orígenes de la lingüística occidental. En el ámbito de la sintaxis es donde esta dicotomía se ha mantenido a lo largo de los siglos con mayor nitidez y rendimiento (piénsese, por ejemplo, en la oposición sustantivo/SUJETO). Su carácter básico no ha impedido, sin embargo, que haya sido punto de disensión entre los diferentes modelos de análisis (gramáticas de dependencias, gramáticas de constituyentes, gramáticas constitutivo-funcionales, etc.), como más abajo se verá.

Por el contrario, en los estudios estrictamente morfológicos esta distinción apenas se ha tenido en cuenta. De esta manera, conceptos como raíz o lexema, entre otros, se han definido y manejado de manera imprecisa, lo que ha supuesto en ocasiones problemas de análisis. La aplicación de esta doble perspectiva al estudio morfológico permitirá enfrentar desde presupuestos divergentes algunos de esos aspectos conflictivos en la morfología del español y ofrecer soluciones diferentes a los mismos (los interfijos, los morfos vacíos, transposiciones morfológicas, etc.).

Este será el objeto de nuestra investigación, cuya importancia radica en la propuesta de extender las posibilidades del análisis sintáctico al nivel morfológico, tarea que pondrá de relieve el carácter unitario de las lenguas y acentuará las relaciones entre los distintos niveles de análisis, especialmente el morfológico y sintáctico, al menos en el marco de una gramática constitutivo-funcional ${ }^{1}$.

\section{EL MARCO TEÓRICO. CLASES Y FUNCIONES EN SINTAXIS}

Este trabajo se enfoca en el marco del funcionalismo europeo, más concretamente en el marco de lo que comúnmente se denomina la Escuela de Santiago (Rojo y Jiménez 1989), modelo constitutivo-funcional, uno de cuyos principios metodológicos se fundamenta en la dicotomía básica señalada:

Las clases de unidades se identifican por la posesión de una característica interna común: fonemas, morfemas, palabras, frases, cláusulas, etc., son clases de unidades, cada una caracterizada por diferentes propiedades, por las que se puede decir que niñ- es un morfema; niños, una palabra (subclase sustantivo); los niños, una frase nominal, y los niños juegan, una cláusula.

Las relaciones (y, más específicamente, las funciones), por el contrario, corresponden a un punto de vista externo (información contextual). Una unidad se relaciona con otras con respecto a las cuales mantiene cierto tipo de relación sintagmática. No tendrá sentido, por tanto, inquirir la función sintáctica de la unidad los niños sin tener en cuenta la cadena de la que forma parte.

1 El estudio de las relaciones entre la morfología y los otros componentes de la gramática es una de las cuestiones más tratadas en la invesigación morfológica. En este apartado, las relaciones con la sintaxis ocupan un lugar destacado (Varela Ortega 1990: 137-192; Piera y Varela 1999; Aguirre 2013: 295-318; Fábregas 2013: 73-86, etc.), en el que desamos hacer una nueva aportación. 
Dada la importancia de estos dos puntos de vista, en este marco teórico se utiliza la convención tipográfica de representar las clases en letras minúsculas en tanto que las funciones se señalan con mayúsculas (figura 1):

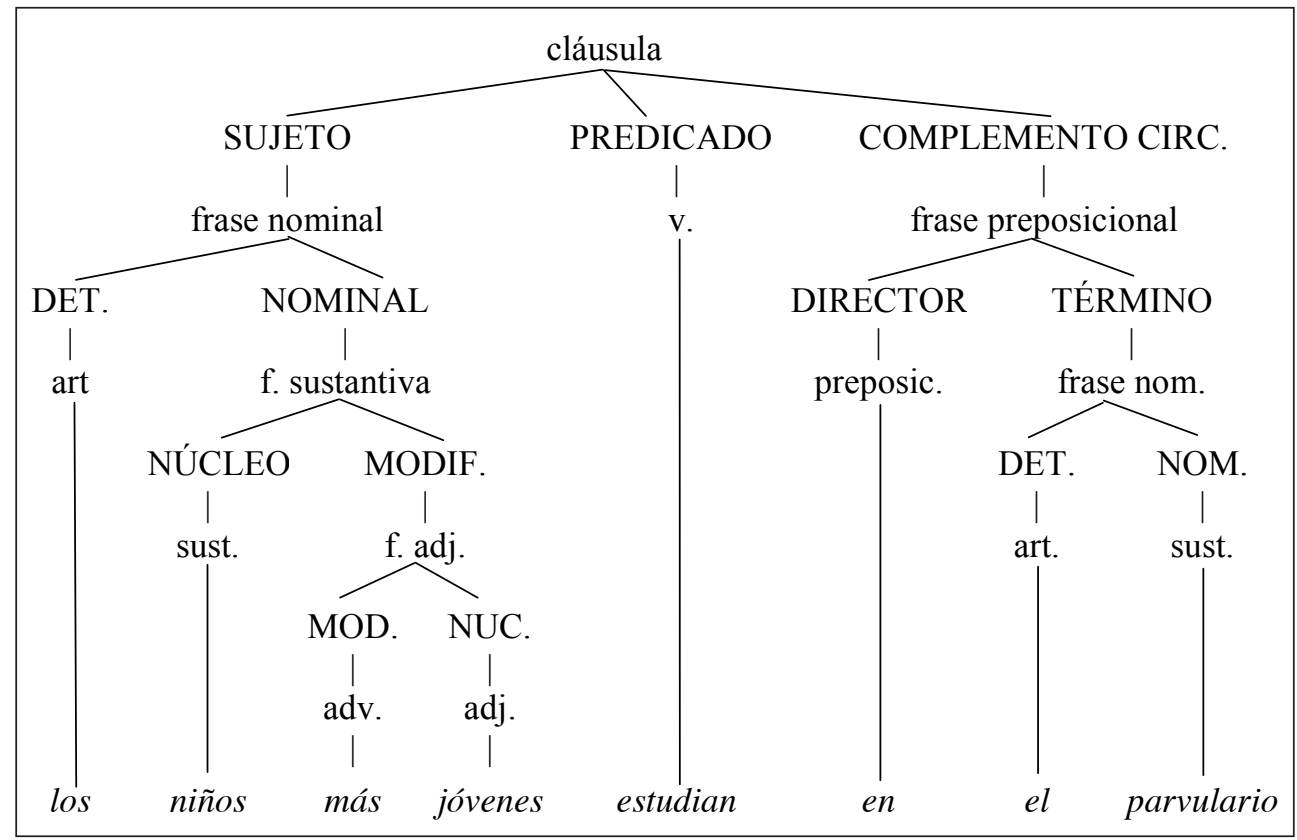

Figura 1. Clases y funciones según Rojo y Jiménez (1989: 54).

Aunque muchas escuelas lingüísticas admiten esta dicotomía fundamental, es sabido que hay una considerable diferencia de perspectiva a la hora de determinar su importancia. El modelo que seguimos, que parte de la relativa independencia entre clases y funciones, en el sentido de que no hay una relación biunívoca entre ellas (Rojo y Jiménez 1989: 22-23), es deudor en gran medida de otras propuestas funcionalistas: tagmémica, gramática sistémica, Longacre, Pike, Dik, Halliday, Hudson, S. Dik, Kuno, etc. (Rojo y Jiménez 1989: 23).

Para no dilatar mucho esta presentación, cabe destacar que otras corrientes funcionalistas y formalistas se oponen drásticamente a este planteamiento. Así, la escuela funcionalista de Oviedo (gramática de dependencias) parte de la prioridad de las funciones sobre las clases y de ahí el recurso constante a la transposición ${ }^{2}$ :

2 Por ejemplo, la función de sujeto es categorialmente sustantiva, por lo que solo podrá aparecer otro tipo de unidad en esa posición si previamente se ha sustantivado, es decir, se ha convertido en sustantivo. Evidentemente, este tipo de gramática funcional no considera que todas las funciones se asocien con una categoría determinada: habrá funciones nominales, adjetivas, adverbiales y otras, como la de atributo o aditamento, que admiten variación en este aspecto (Gutiérrez Ordóñez 1997: 158-160). 
En las gramáticas funcionales se parte de conceptos tales como sujeto, implemento, suplemento, etc. Aunque también se apela a categorías (nombre, verbo, adverbio), estas son conceptos derivados de las funciones (Gutiérrez Ordóñez 1991: 4).

Mientras que en una gramática de este corte "El concepto de categoría es derivado del concepto de función (teóricamente preeminente)" (Gutiérrez Ordóñez 1991: 6), en las gramáticas constitutivas, entre ellas la gramática generativa, por el contrario, la perspectiva es la opuesta: no encontraremos categorías como sujeto sino SN, SV, SP, Sadj, Sadv... Para este conjunto de gramáticas las funciones no son primitivos lingüísticos sino relaciones derivadas:

Algunas teorías lingüísticas consideran que las funciones (sujeto, objeto directo, etc.) son conceptos primitivos de la sintaxis. En otros enfoques, por el contrario, se las trata como nociones relacionales derivadas de la estructura de las oraciones. En esta obra, concebiremos las funciones como resultado de la relación entre los constituyentes que integran una oración (Hernanz y Brucart 1987: 36-37)33.

\section{METODOLOGÍA}

Desde el último cuarto de siglo pasado se han producido considerables avances en el análisis de la estructura de la palabra, principalmente en el marco de la gramática generativa (algunos hitos: Aronoff 1976, Scalise 1984) o de otros modelos teóricos, como el cognitivo (Bybee 1985), de los que son deudores multitud de estudios aplicados al análisis morfológico del español.

Para contrastar las posibilidades del modelo constitutivo-funcional en el nivel morfológico hemos procedido, pues, a la consulta de las principales obras de referencia aparecidas en los últimos años en español (principalmente manuales universitarios) junto con algunos tratados clásicos en la materia (vid. Referencias). De este modo, analizaremos cómo son tratados algunos aspectos problemáticos del análisis morfológico y cómo se pueden enfocar desde nuestra perspectiva.

En líneas generales, podemos constatar que esta doble aproximación es desconocida en estos estudios, en coherencia con el carácter constitutivo de los modelos generativos. Más llamativo es que los modelos de carácter funcionalista, como el que adoptamos, tampoco hayan explorado esta posibilidad. Sin embargo, es evidente que, si hay palabras de estructura compleja, si la palabra es un todo en el que se articulan diferentes partes (sintagma), en su interior tienen que establecerse distintos tipos de relaciones, entre ellas las funcionales. Por todo ello, consideramos que también en el nivel morfológico debemos mantener esta importante doble perspectiva de análisis si pretendemos solventar algunas confusiones que con frecuencia se producen.

3 En concreto, especifican estos autores: "Un modo de establecer formalmente la diferencia entre las distintas funciones consiste en definirlas como el resultado de la relación que se establece entre la categoría que representa a cada una de ellas [...] y la primera proyección que domina a aquélla. De este modo, el sujeto quedará caracterizado como el nudo SN dominado inmediatamente por O (sujeto = [SN, O])” (Hernanz y Brucart 1987: 37). 


\section{UNIDADES Y FUNCIONES EN MORFOLOGÍA}

\subsection{Raíz vs. lexema}

Nos referimos, por ejemplo, a la distinción entre conceptos como RAÍZ y lexema ${ }^{4}$. Con frecuencia estos conceptos se usan como sinónimos (de ahí que a menudo se hable de la raiz o lexema de una palabra):

Una raíz o lexema como nac- puede constituirse en palabra con solo recibir las marcas de flexión apropiadas (Varela Ortega 2005: 18).

Los lexemas o raíces son los únicos capaces por sí mismos de integrar una palabra (Aguirre 2013: 29).

La raíz y el radical han sino denominados lexema por los estructuralistas europeos y morfema léxico en la lingüística americana (Otaola 2004: 95).

Está claro que, si se trata de meros sinónimos, uno de ellos es prescindible, y es eso lo que sucede con frecuencia. Así, Fábregas (2013:38-39) opta por el término raíz en detrimento del más impreciso de lexema.

Este autor, en su completo manual, parte de una primera distinción entre lexemas y afijos que, finalmente, será desechada, al sustituir el impreciso concepto de lexema por el de raíz. En la primera aproximación, lexema y afijo se contraponen en palabras como trabaja-dor:

El morfema trabaja- puede aparecer solo en un enunciado, como ¡Trabaja! En cambio, el morfema -dor, no. El significado del morfema trabaja parece estable: expresa una acción determinada; por el contrario, el significado de -dor es más abstracto [...]. Los morfemas que tienen las propiedades de trabaja se conocen como LEXEMAS, mientras que los que se comportan como -dor se conocen como AFIJOS. Algunos autores utilizan el término RAÍZ para hablar de los lexemas, pero en este manual los diferenciaremos (Fábregas 2013: 21-22).

Como podemos apreciar, lexema en esta caracterización primera equivale a lo que generalmente denominamos tema morfológico. Por oposición, la raíz quedará definida de la siguiente manera:

Llamamos a cant- y al resto de elementos que expresen conceptos y puedan combinarse con afijos para formar palabras RAÍZ. La raíz normalmente no puede aparecer sola, al contrario de lo que llamamos, de forma vaga, lexema (Fábregas 2013: 39).

4 Evidentemente, nos referimos aquí al uso de lexema como 'morfema léxico'. Siguiendo la tradición anglosajona (Lyons 1968: $§ 5.4 .4$ ), en ocasiones lexema (lexeme) se utiliza con el valor de lema (forma que engloba las variantes flexivas de una unidad): "So we have to make a distinction between the notion 'word' in an abstract sense (lexeme) and the notion 'word' in the sense of 'concrete word as used in a sentence'. The concrete words walk, walks, walked, and walking can be qualified as word forms of the lexeme WALK" (Booij 2005: 3). En la misma línea, dice Aguirre (2013) que ante río y ríos nos hallamos ante dos palabras que son, de algún modo, la misma. Concluye: "Lo que sucede es que nos encontramos ante dos formas de un mismo lexema" (Aguirre 2013: 23) y más adelante: "Un mismo lexema puede presentar distintas formas según el contexto sintáctico en el que aparezca y los significados de tipo gramatical que se añadan al significado léxico de la palabra" (Aguirre 2013: 217). Con todo, a lo largo de esta obra predomina el término lexema con su valor específico de sinónimo de raíz (Aguirre 2013: 29, etc.). 
Resumamos las unidades que hemos identificado hasta ahora, para entender dónde estamos ahora que hemos eliminado de nuestro vocabulario el término 'lexema'. Tenemos dos. Por una parte están los afijos, que aportan información de muy distintos tipos, como el género, la clase léxica a la que pertenece una palabra o la concordancia [...]. Por otra parte, están las raíces, que expresan conceptos del mundo real (Fábregas 2013: 39).

En esquema, la oposición lexema/afijo es sustituida por raíz/afijo, al carecer de sentido la distinción lexema/raíz (el lexema es fruto de un análisis inconcluso trabaja-, el cual, una vez completado en trabaj- $a$, permitirá diferenciar la raíz).

En la misma línea, también dentro del marco generativo, Varela Ortega (1990) identifica los siguientes primitivos en el componente morfológico: palabra simple, tema, afijo, raíz. Obsérvese la ausencia del lexema:

La morfología constituye, como hemos dicho, un componente autónomo que consta de sus propios primitivos o formantes, los cuales se identifican, en su mayor parte, con una terminología propia de este componente. Estos son: palabra simple, tema, afijo, raíz (Varela Ortega 1990: 32).

Otros autores que han elaborado material en forma de manuales o tratados universitarios ofrecen, en cambio, una distinción entre estos dos términos. En una obra posterior, la propia Varela Ortega (2005) diferencia raíz y lexema de la siguiente manera:

La unidad básica de la palabra se denomina 'raíz' desde el punto de vista formal o del 'significante', y 'lexema' -o 'semantema'- desde el punto de vista semántico o del 'significado' (Varela Ortega 2005: 17, n. 1).

Es decir, si quisiéramos representar esta distinción atendiendo a la convención saussureana nos encontraríamos ante un signo lingüístico representable de la siguiente manera (figura 2):

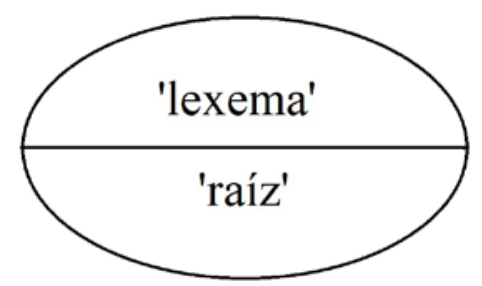

Figura 2. Raíz, lexema y los dos planos de un signo.

La influencia de Varela Ortega es palpable en Aguirre (2013), que acepta la misma distinción relativa a los dos planos del signo lingüístico:

La raíz o lexema es el morfema más primario, el último constituyente de la palabra, el que nos queda cuando hemos quitado todos los afijos. Es el que aporta el significado léxico de la palabra. Desde el punto de vista semántico o del significado le llamamos lexema y desde el punto de vista formal, raíz (Aguirre 2013: 29). 
La causa de esta confusión de conceptos y de la consiguiente supresión de uno de ellos (lexema) o de su redefinición obedece, en nuestra opinión, a la mezcla de criterios (internos y externos) a la hora de identificar las unidades lingüísticas. Es esta una limitación que ha caracterizado a la tradición lingüística desde antiguo (otras muchas unidades se han definido mezclando indiscriminadamente estas perspectivas, entre ellas la oración ${ }^{5}$ ). Sin embargo, si se delimitan adecuadamente los dos puntos de vista de que estamos hablando, se solventarán -creemos- algunas de las incoherencias que caracterizan los estudios morfológicos.

En efecto, si atendemos a las principales definiciones que acabamos de ver del término raíz, y otras que podamos traer a colación, observaremos que en ellas se utilizan rasgos o características pertenecientes a diferentes ámbitos:

- Semántico: las raíces “expresan conceptos del mundo real” (Fábregas 2013: 39), “aporta[n] el significado léxico de la palabra” (Aguirre 2013: 29). Este es el criterio principal para definir la raíz en la $N G L E$ : "Tanto en morfología flexiva como en la léxica se distinguen la RAíz (es decir, el segmento de la palabra que aporta el significado léxico) y los AFIJOS, que agregan a esta informaciones de diverso tipo" (NGLE 2009: 22).

- Distribucional: "La raíz normalmente no puede aparecer sola" (Fábregas 2013: 39), "es una forma necesariamente ligada" (Varela Ortega 1999: 34).

- Funcional: "Por raíz se entiende la primera base, el elemento nuclear del que parte la primera operación morfológica" (Varela Ortega 1999: 34), "se trata del segmento básico y constante en el significante de cualquier palabra" (Pena 1999: 4315)6, "el último constituyente de la palabra, el que nos queda cuando hemos quitado todos los afijos (Aguirre 2013: 29) .

Esta coincidencia de rasgos en una misma entidad es la que hace innecesario incluir al lexema en un estudio morfológico: Si todas las raíces presentan significado léxico, es evidente que el concepto de lexema 'morfema léxico' resulta sobrante. Si todas las raíces son lexemas se podrán relegar estos del listado de clases de unidades, por redundantes (como se procede en un análisis funcionalista que dé prioridad a las funciones). Ocurre, sin embargo, que esta aserción ("todas las raíces son léxicas”) necesita una comprobación empírica.

5 Como es sabido, la oración se ha definido desde el punto de vista externo como la 'unidad del habla' (es decir, se tiene en cuenta su capacidad funcional de actuar como enunciado), mientras que, desde el punto de vista interno, constitutivo, se concibe como una construcción formada por dos constituyentes (sujeto y predicado) o, en otras interpretaciones, constituida por un predicado como núcleo. Como ambos criterios no coinciden (pertenecen a ejes distintos) habrá oraciones desde el punto de vista constitutivo (quien diga eso) que no lo serán desde el punto de vista de su funcionamiento en el habla (oraciones gramaticales o proposiciones). Y, a la inversa, habrá cadenas con 'sentido completo' carentes de las propiedades constitutivas (las oraciones psicológicas del tipo ¡la hora!) (Hernanz y Brucart 1987: 48-56; Rojo y Jiménez 1989: 97-100).

6 La cita continúa así "Se trata del segmento básico y constante en el significante de cualquier palabra que, como resultado de eliminar en tales significantes todos los afijos derivativos y/o flexivos, es irreductible o no susceptible de ulterior análisis o, desde otra perspectiva, la unidad que constituye el punto de partida de cualquier construcción morfológica" (Pena 1999: 4315).

7 Todas estas características suelen aparecer juntas en una misma definición: "Con el término raíz se denomina el morfema ligado que constituye el inicio de una construcción morfológica (p. ej. sol- en el adjetivo denominal sol-ar). Se trata del segmento formal compartido por todos los miembros de una familia léxica, a los que aporta el significado léxico común” (Felíu Arquiola 2009: 55). 
Sucede, en efecto, que en ocasiones funciones y clases mantienen ciertas relaciones de solidaridad, que se pueden cuantificar de manera estadística. Más arriba hemos defendido que la relación entre funciones y clases no es biunívoca, pero ello no implica que sea totalmente aleatoria. En este punto, cada lengua natural se comporta de muy diferente manera. Pudiera pensarse pues que, aun aceptando la radical distinción entre funciones y clases como principio organizador del análisis lingüístico, concluyéramos que en el caso que nos ocupa raíces y lexemas son siempre solidarios.

Antes de seguir con nuestra argumentación convendrá, sin embargo, que definamos al lexema para clarificar nuestro punto de vista. Como es general en la mayoría de los tratados gramaticales, entendemos por lexema un 'morfema léxico', es decir, una 'unidad mínima con significado léxico' (Bosque 1983: 117-118). No es este el momento ni el lugar para que nos detengamos en la diferencia entre unidades léxicas y unidades gramaticales, distinción bien asentada en los estudios lingüísticos a pesar de sus límites imprecisos. Bástenos señalar que las unidades léxicas -más allá de caracterizarse por cierto significado descriptivo que, con frecuencia, comparten con unidades gramaticales- se diferencian por su organización en paradigmas abiertos.

\subsection{Raíz-afijo vs. lexema-derivativo-flexivo}

Partiendo de estos postulados es insostenible que la posición radical esté ocupada necesariamente por un lexema. De la misma manera, también es posible que en posición afijal aparezca un morfema léxico. Esto es lo que podemos deducir en Varela Ortega (2005: 1718), cuando diferencia entre afijos léxicos (re-en renacer) y afijos gramaticales (es decir, sin significado léxico, como la vocal temática -e- y el sufijo flexivo - $r$ de renacer).

Si interpretamos bien a Varela Ortega (2005: 17-18), son morfemas léxicos por su contenido tanto los 'lexemas' (ligados o libres: nac- en renacer, mar, pan, etc.) como los afijos derivativos (re- en renacer), por oposición a los gramaticales (los flexivos). Su punto de partida es el significado ${ }^{8}$. Si adoptamos como criterio clasificador solo el significado, la situación de los morfemas derivativos se vuelve muy compleja (la mayoría de los autores no los consideran lexemas sino que para unos ocuparían una situación intermedia entre las unidades léxicas y las gramaticales y para otros serían morfemas plenamente gramaticales, en todo caso dentro

8 Más adelante aclara: "Los afijos que contribuyen a la formación de nuevas palabras son aquellos que tienen contenido léxico y que, por su capacidad de derivar otras formas léxicas, se denominan 'afijos derivativos'. Los que simplemente transmiten contenidos gramaticales se denominan 'afijos flexivos' y no contribuyen a formar nuevas palabras sino a flexionarlas" (Varela Ortega 2005: 18). Esta postura, que identifica en el significado a lexemas y morfemas derivativos, se asemeja considerablemente a la sostenida, en el campo opuesto de la lingüística funcionalista, por Coseriu (1977: 140; 1978: 248). Este autor considera que el procedimiento que en sentido estricto denomina derivación (derivación heterogénea) desde el punto de vista del significado se asemeja a la composición: "La derivación [...] es una combinación de dos lexemas (pertenecientes normalmente a campos diferentes), de los que uno (el que se presenta en el significante) determina al otro (que está representado en el significante por el sufijo derivativo o por cero) [...]. Las «palabras derivadas» son sintagmas de contenido con expresión sintética. Desde este punto de vista, lo que se llama «composición» no es muchas veces sino una derivación «explicada» (es decir explícita) o, mejor, una síntesis sintagmática de grado inferior al de la derivación” (Coseriu 1977: 140). Nótese, sin embargo, que Coseriu diferencia entre el significado y el significante. Las semejanzas se producen sólo en el significado. Sin embargo, las categorías en la gramática funcional surgen de la asociación significado-significante, lo cual implica, en nuestra opinión, que, como categorías lingüísticas (asociación significado/significante), la composición y la derivación no se pueden equiparar ni, consecuentemente, los lexemas y los morfemas derivativos. 
de una gradación en la que ocuparían la posición más alejada de los prototípicamente gramaticales: por este orden flexivos-apreciativos-derivativos). En este sentido, recordemos como I. Bosque (1983: 118) incide en el valor gramatical de estas unidades al señalar que con los morfemas derivativos se produce una gramaticalización de los significados léxicos 9 .

Sea como fuere e, independientemente de donde establezcamos el límite entre lo léxico y lo gramatical, lo cierto es que al hablar de lexemas (o morfemas léxicos), morfemas derivativos, morfemas apreciativos y morfemas flexivos sin duda nos estamos refiriendo a clases de unidades, caracterizadas por la posesión de una serie de propiedades que las diferencian entre sí, propiedades que los gramáticos han descrito exhaustivamente (Bosque 1983: 133-135; Varela Ortega 2005: 33; Fábregas 2013: 177-180, etc.). Por ello, cuando se diferencian estos tipos de morfemas en los tratados morfológicos se suele recurrir al criterio denominado de la naturaleza gramatical (Bosque 1983: 133). Por naturaleza gramatical debe entenderse lo que aquí denominamos punto de vista interno, características inherentes.

Por tanto, aquellas definiciones que aluden a la naturaleza léxica de la raíz propiamente hacen referencia a la unidad que denominamos lexema. Quedan, consecuentemente, como rasgos definitorios de la raíz aquellos otros que aluden a su naturaleza funcional (núcleo de la palabra, primera base, elemento constante, etc., no todos de la misma validez ${ }^{10}$ ). Y, en este punto, el correlato de raíz es afijo y el criterio que se utiliza para dar cuenta de esta distinción ya no es la naturaleza gramatical sino el "criterio distribucional" (Pena 1999: 4325-4326) o la "posición en la palabra" (Bosque 1983: 133). Es lo que denominamos punto de vista externo (por su relación con las otras unidades del todo).

Dicho de otra manera, RAÍZ es a SUJETO, como lexema a sustantivo si se nos permite este sencillo paralelismo que podemos completar en el siguiente esquema (tabla 1):

\begin{tabular}{|c|l|}
\hline $\begin{array}{c}\text { Punto de vista externo } \\
\text { (funcional, } \\
\text { distribucional) }\end{array}$ & RAÍZ \\
\cline { 2 - 2 } & AFIJO (PREFIJO, SUFIJO, INTERFIJO, INFIJO, CIRCUNFIJO) \\
\cline { 2 - 2 } $\begin{array}{c}\text { Punto de vista interno } \\
\text { (características } \\
\text { intrínsecas) }\end{array}$ & Morfema léxico o lexema \\
\cline { 2 - 2 } & Morfema gramatical (derivativo, apreciativo, flexivo) \\
\cline { 2 - 2 } & Morfo vacio, elemento temático (vocal temática en castellano) \\
\hline
\end{tabular}

Tabla 1. Funciones y clases morfológicas.

\footnotetext{
9 Por tanto, la diferencia entre morfemas derivativos y morfemas léxicos no se fundamenta tanto en el tipo de significado (sustancia del contenido) cuanto en la organización de esas unidades en una lengua determinada. Con frecuencia se mencionan significados que pueden ser expresados mediante procedimientos léxicos o derivativos (así, por ejemplo, en tienda de bebidas, bodega, vinacoteca o cervecería, hay un sema común asociado al morfema derivativo -ería, al elemento grecolatino -teca, al lexema tienda y, de una manera semánticamente más compleja, al lexema bodega).

10 Alguno, como el de "elemento constante en una familia léxica", no deja de ser circular. Piénsese que en series como carnicero, peluquero, enfermero, etc. el elemento constante es -er-. Este es el que los agrupa semánticamente en un campo léxico (Coseriu: 1977: 135, 140).

11 En efecto, la distinción libre/ligado es de naturaleza distribucional: una misma unidad puede, en principio, aparecer como segmento libre (sol, cerca, ven) o ligado (sol-ar, cerqu-ita, ven-i-d), lo cual no impide que estadísticamente haya clases que suelen preferir la distribución libre (preposiciones, adverbios, conjunciones) y otras, la ligada (verbos).
} 
En otros términos, la anterior distinción se podría traducir en términos sintácticos diciendo que PREFIJO, RAÍZ, INTERFIJO o SUFIJO son casillas funcionales (slots) de la palabra que pueden ser cubiertas por unidades (fillers) tales como lexemas, morfemas derivativos, morfemas apreciativos o morfemas flexivos, de la misma manera que en una cláusula hallamos las casillas funcionales de SUJETO, CD, RÉGIMEN, CI, etc. que saturan palabras, frases, otras cláusulas, etc.

Esta concepción que acabamos de defender se puede rastrear implícitamente, aunque sin desarrollo, en el análisis de alguna de las obras de referencia que hemos utilizado en nuestra exposición:

Las reglas [de formación de palabras] van a utilizar un elemento de base con significado léxico independiente, un lexema que va a funcionar como raíz de la palabra [cursiva nuestra], y a esa base le vamos a añadir otros componentes" (Aguirre 2013: 57).

\section{RENDIMIENTO DE LA OPOSICIÓN}

\subsection{Correspondencia clases-funciones morfológicas}

Establecida esta distinción que implica trasladar al ámbito morfológico el doble análisis que muchos funcionalistas aplicamos en el nivel sintáctico, la parte final de este trabajo se centrará en determinar hasta qué punto es útil este trasvase. Si, como hemos sugerido, en el nivel morfológico la solidaridad entre clases y funciones es prácticamente general (si todas las raíces son léxicas, todos los prefijos, derivatios, todos los morfemas flexivos aparecen en posición sufijal...), la distinción que planteamos no superaría en mucho los límites de una mera corrección terminológica que conduciría a un análisis en gran medida redundante. $\mathrm{Si}$, por el contrario, esta solidaridad no es total, serán los estudios empíricos en cada lengua los que determinarán su trascendencia de acuerdo con su capacidad para resolver incoherencias y análisis discutibles.

Evidentemente, hay argumentos para sostener que la mencionada solidaridad total no se produce, aunque el condicionamiento mutuo clase-función sea mayor en el nivel morfológico que en el sintáctico. Veamos algunas muestras para ilustrar el rendimiento de la oposición:

Del carácter complementario de la distinción RAÍZ/lexema se deduce la posibilidad de que al lado de palabras cuya raíz esté ocupada por morfemas léxicos (niñ-er-o-s, lleg-a-r) puedan existir otras en cuya posición nuclear aparezcan morfemas gramaticales. Aquí figurarán todas las palabras gramaticales complejas, cuyo núcleo es, necesariamente, un morfema gramatical ( $l-a-s$, ell-a-s, est-a-s, etc., donde los morfemas $l$-, ell-, est- no pueden ser calificados de lexemas si aceptamos la distinción generalmente establecida entre unidades léxicas y gramaticales) ${ }^{12}$ :

12 Obsérvese que para este tipo de morfema gramatical no existe una denominación precisa. Términos como morfema (Martinet), gramema (Pottier) y otros que podemos comprobar en Bosque (1983: 118) tienen un valor general (por oposición a lexema o morfema léxico), que incluiría como subtipo (junto a flexivos y derivativos) a los que aquí nos interesan. A falta de un término más adecuado usaremos morfema gramatical en un doble sentido: general (opuesto a léxico) y específico (por oposición a flexivos, apreciativos y derivativos). 

(1) RAÍZ
RAÍZ
m. lex.
m. gr.

En las siguientes representaciones (en las que se marca siguiendo la convención apuntada las funciones con letras capitales y las clases en minúsculas) se puede apreciar la diferencia:

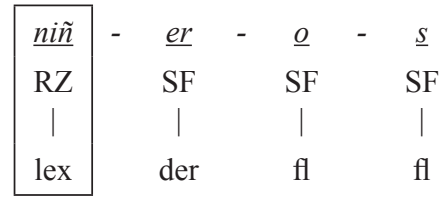

(2b)

\begin{tabular}{|c|c|c|}
\hline ell & $-\quad \underline{a}$ & $\underline{S}$ \\
\hline RZ & SF & SF \\
\hline 1 & | & | \\
\hline mgr & $\mathrm{fl}$ & fl \\
\hline
\end{tabular}

\subsection{Interfijos}

Los interfijos constituyen una de esas entidades lingüísticas sobre las que mayor discusión ha habido en nuestra tradición gramatical moderna (Portolés 1999, Camacho 2002, Fábregas 2017, etc.), sobre todo en lo referente a si carecen necesariamente de significado.

Desde nuestra perspectiva estas cuestiones carecen de relevancia: la posición de INTERFIJO conforma una casilla funcional potencial de una palabra. Esta casilla podrá ser rellenada con unidades carentes de significado (morfos vacios), lo que sucede frecuentemente, pero también con unidades significativas (lo que denominaríamos morfemas llenos, si esta expresión no resultase redundante $\left.{ }^{13}\right)$. De esta manera, en hum-ar-eda ocupa la posición de interfijo un morfo vacío (-ar-) en tanto que en tont-orr-ón, por ejemplo, el interfijo es un morfema apreciativo (-orr-):
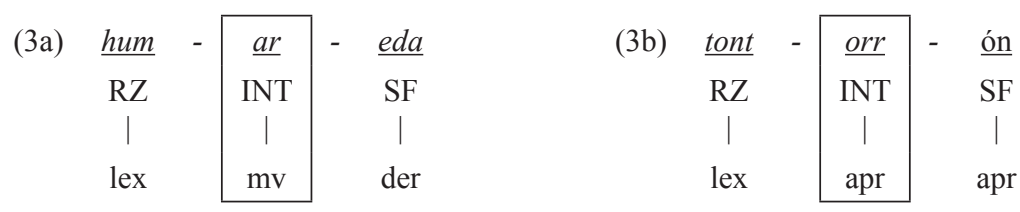

Desde la perspectiva contraria, nos podemos plantear si la unidad -orr- que acabamos de mencionar (y sus variantes -arr-, -urr-, -irr-) ha de ocupar necesariamente una posición de interfijo, es decir, si es un interfijo. De acuerdo con nuestros presupuestos, eso no ha de suceder necesariamente. Y, en efecto, el análisis del léxico español muestra como este morfema apreciativo puede funcionar como INTERFIJO o como SUFIJO, según se comprueba en la siguiente relación ${ }^{14}$ y representación $(4 a)-(4 b)$ :

13 No es redundante si se acepta que un morfema es simplemente un 'segmento morfológico' (NGLE, $\S 1.5 \mathrm{~d})$. 14 Siguiendo la practica tradicional, los morfemas flexivos no entran en consideración a la hora de diferenciar sufijos e interfijos: El punto de partida de esta distinción es el tema de la palabra ("la unidad que resulta de restar los afijos flexivo", Pena 1999: 4315) y no la palabra misma. Por tanto, es común admitir que -orr-o -it- funcionan de sufijos en perr-it-o o ti-orr-o, a pesar de que nunca pueden aparecer a final de palabra (*perrit, *tior), porque sí pueden situarse en el final del tema, que es donde se adjuntan los flexivos ([[ perr $\left._{\text {RAiz }}\right]$-it $\left.{ }_{\text {TEMA }}\right]$-o $]$ ). Este comportamiento está determinado por el hecho de que ambos morfemas son transparentes con respecto al género, lo cual implica que este se materializa necesariamente después de ellos, aunque no esté presente en la base (mes $>$ mes-ec-it-o, caliente $>$ calent-orr-o/-a, vida $>$ vid-orr-a). Con todo, la casuística con respecto a estas formaciones es muy compleja, como se puede comprobar en Lázaro Mora (1999). 
INTERFIJO (situación medial en el tema): tont-orr-ón, sant-urr-ón, vent-arr-ón, chiqu-irr-itin, dulz-arr-ón, chic-arr-ón, sec-arr-al, mans-urr-ón, etc.

SufiJo (posición final del tema): tint-orr-o, ti-orr-o/-a, mach-orr-o/-a, abej-orr-o, chin-arr$o$, calent-orr-o/-a,vid-orr-a, etc.

\begin{tabular}{|c|c|c|}
\hline (4a) & tont - & orr \\
\hline & RZ & INT \\
\hline & 1 & $\mid$ \\
\hline & lex & apr \\
\hline
\end{tabular}

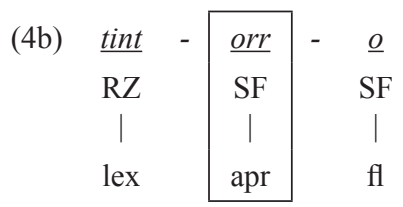

De esta manera solucionamos una cuestión que debate Portolés (1999: 5047):

En tal caso, si mantuviéramos el concepto de sufijo verbal para nuestros interfijos, se debería distinguir entre un interfijo -ot- en bail-ot-e-ar, pic-ot-e-ar y pis-ot-e-ar, y un sufijo verbal -ot- en barb-ot-ar, o entre un interfijo -urr- en cant-urr-e-a-r y chap-urr-e-ar, y un sufijo verbal en despach-urr-ar y desapanz-urr-ar, o, incluso, entre un interfijo -uk- en bes-uqu-e-ar y un sufijo -uk-en bes-uc-ar. Evidentemente, una descripción de este tipo sería enormemente enojosa.

En nuestra opinión, ante este segmento /ot/ no tiene sentido plantearse si es un sufijo o un interfijo: es un morfema apreciativo que puede funcionar como sufijo (amig-ot-e) o como interfijo (pis-ot-e-a-r, man-ot-azo). Por otra parte, este proceder en absoluto es enojoso (pues no aumenta el número de unidades o funciones, simplemente se obtiene el máximo rendimiento de las ya establecidas, como no es enojoso en sintaxis señalar que niños, siendo siempre un sustantivo, funciona como suJETo en no vinieron niños y como $\mathrm{CD}$ en no había niños).

\subsection{Diminutivos}

Se plantean Lázaro Mora (1999: 4658-4662) y Aguirre (2013: 283-284), a propósito de casos como Osquitar o azuquitar, si los morfemas diminutivos son infijos o sufijos:

Si consideramos que el morfema de diminutivo es un infijo que se intercala en la raíz o lexema de la palabra, explicamos muy bien algunas formaciones de diminutivos como $a z u ́-$ car $>$ azuquitar, que quedaría segmentada considerando el morfema -it- como un infijo: azuqu-it-ar

La extensión de este análisis a la generalidad de los diminutivos queda descartada por la mayor parte de los lingüistas (Aguirre 2013:283-284).

Evidentemente, esta extensión queda descartada porque en la inmensa mayoría de los casos los diminutivos actúan como sufijos (pequeñ-it-a), es decir, ocupan la posición final del tema, antes de la inserción de los flexivos. Lo que cabe concluir es, pues, que los morfemas pertenecientes a la subclase de los diminutivos ocupan generalmente una posición de sufijos y, ocasionalmente, otras como la de infijos (azuquítar $)^{15}$.

15 Aguirre concluye coherentemente "Podemos concluir que el proceso de diminutivización no es del todo homogéneo. En la mayoría de los casos estaríamos ante un proceso normal de sufijación (...). Pero en algunos casos encontramos formaciones en las que el diminutivo aparece infijado" (Aguirre 2013: 284). 
En la misma línea, en un trabajo reciente, Fábregas (2017) aporta un considerable número de argumentos en favor de la opinión de Lázaro Mora (1999) de que ciertos morfemas apreciativos verbales presentan las mismas propiedades que los interfijos (resquebr-aj-ar / pequeñ-aj-o, apret-uj-ar / bland-uj-o, dorm-it-ar / gat-it-o, bail-ot-ear / gand-ot-e, etc.). Su conclusión apunta en la línea de considerar la identidad categorial de ambas categorías: "los interfijos y los morfemas apreciativos son en realidad la misma clase de objetos" (Fábregas 2017: 135). Desde nuestra perspectiva estas similitudes son las esperables, no porque se produzca una identidad categorial entre ambos grupos sino porque en realidad se están analizando las mismas unidades desde la doble perspectiva de su distribución en la palabra y de su naturaleza intrínseca de morfemas apreciativos, como se ha señalado en el apartado anterior.

\subsection{Metábasis o transposición}

Otra cuestión morfológica que podemos tratar desde esta perspectiva es la relativa a la metábasis o transposición, entendida como un cambio de clase. Este mecanismo es básico en aproximaciones como la que partiendo de Ch. Bally y continuada por L. Tesnière florece en la escuela fundada por E. Alarcos (Gutiérrez Ordóñez 1997:123-160). En otros planteamientos de índole también funcionalista, como la que defendemos, este recurso debe quedar limitado a los casos realmente comprobados de cambio categorial, pues, si partimos del supuesto de que clases y funciones no mantienen una relación biunívoca, el hecho de que un determinado elemento aparezca en una casilla que a priori no le corresponde no presupone un cambio de clase: así, una cláusula con núcleo infinitivo seguirá siendo cláusula y su núcleo un verbo aunque desempeñe la función de sujeto u objeto: el lamentarse dulcemente dos pastores. Solo hablaremos de transposición (sustantivación en este caso) cuando realmente el infinitivo se ha convertido en un sustantivo (El dulce lamentar de dos pastores).

En segundo lugar, el concepto de transposición se fundamenta, como hemos señalado, en la asociación estable clase-función (funciones sustantivas como la de sujeto, funciones adjetivas como la de modificador de un sustantivo, funciones adverbiales como la de modificador verbal, etc.). En nuestra visión, tales asociaciones se basan en frecuencias estadísticas antes que en propiedades gramaticales, lo cual no implica que todas las combinaciones sean posibles. Ciertamente, en el nivel morfológico estas solidaridades son más frecuentes que en sintaxis. Aun así están lejos de la absoluta rigidez: sabemos que en castellano los morfemas flexivos ocupan siempre posición sufijal (pero esto no deja de ser un accidente si tenemos en cuenta otras lenguas o, incluso, si traemos a colación formas como cualesquiera o los tiempos compuestos verbales - habíamos decidido-, donde los flexivos /es/ y /mos/ aparecen en interior de palabra); los morfemas derivativos y los apreciativos, por el contrario, pueden ocupar una posición prefijal (super-emocionado) o sufijal (emocionad-ísimo).

Partiendo de los anteriores presupuestos, nos podemos plantear hasta qué punto se puede hablar de metábasis entre las unidades morfológicas. La historia de la lengua nos muestra que un morfema léxico puede gramaticalizarse. Sin duda, el más conocido es el morfema derivativo -mente, formador de adverbios, cuyo origen se halla en el lexema homónimo ${ }^{16}$. En la actualidad, la conversión de un lexema en un morfema derivativo se comprueba en el

16 Lo cual justifica las peculiaridades de los adverbios en -mente, con respecto al resto de las palabras derivadas: coordinación de raíces: [[lisa y llana] mente], permanencia del formante de género femenino (plácid-a-mente), doble acentuación. 
término anglosajón -gate (/'geit/), adaptado en castellano como derivativo a partir del cual se forman decenas de palabras (valijagate, piñeragate, cocagate, pemexgate, toallagate, dieselgate, etc.), la mayoría efímeras ${ }^{17}$. Igualmente, un morfema gramatical (flexivo, derivativo o apreciativo) puede lexicalizarse (los ismos, los emas) o cambiar de subclase (como le ha sucedido a -iño,- $a$, diminutivo gallego formado a partir de un derivativo).

Pero no siempre que una unidad desempeña una función que nos parece extraña es necesario recurrir a la metábasis. Pensemos en series léxicas como la siguiente: euroescéptico, eurodiputado, eurotúnel, eurobarómetro, eurocámara, eurogrupo, eurozona e, incluso, eurohuelga, eurodesencanto, eurorrealista... Un posible análisis de estas unidades consiste en considerar que son formaciones compuestas (o más concretamente un subtipo de compuesto, los formados por entrecruzamiento o acronimia). Ahora bien, el entrecruzamiento no es un procedimiento regular: se utiliza ocasionalmente en creaciones intencionadas en las que casi siempre se suprime la parte final de la primera base y la inicial de la segunda utilizando un fonema común como frontera: burkini < burk [a bi]kini). En el caso que nos ocupa, euro es un elemento recurrente. Actúa como un elemento prefijal y, por tanto, podría pensarse en una metábasis morfema léxico $>$ morfema derivativo. Pero no es necesario recurrir a este proceso, la relativa falta de correspondencia clases-funciones nos permite considerar que nos hallamos ante una unidad léxica europa que se realiza con variación alomórfica dependiendo de su función: /europa/ o /europ/ en función de raíz (Europa, europ-e-o) y /euro/ en función prefijal (euro-cámara, euro-diputad-a):

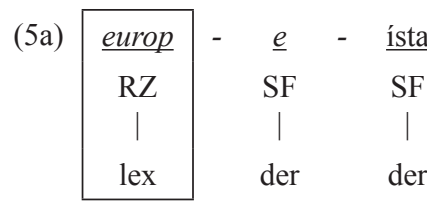

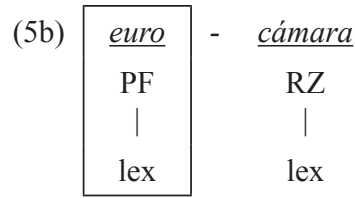

Lo mismo ocurre con otras unidades morfológicas, cual es el caso del derivativo -ble, que aparece bajo esta forma en posición sufijal (visible) y con el alomorfo -bil- cuando ocupa posición de interfijo (visibilidad). No hay metábasis en euro prefijal, pues no se produce un cambio de naturaleza gramatical (de lexema a morfema derivativo) sino una ampliación de funciones.

\section{CONCLUSIONES}

Como resumen conclusivo de lo que acabamos de presentar, podemos señalar los siguientes postulados:

- En el marco de una gramática de orientación constitutivo-funcional, la distinción entre clases de unidades y funciones debe ser ampliada del nivel sintáctico al nivel morfológico.

17 Desde el punto de vista semántico este morfema derivativo alude a actividades que se intentan ocultar, al contrario que los tradicionales - azo y - ada, que nombran eventos que se pretende que provoquen impacto público (compárese sanjuangate y sanjuanada). En ocasiones estos derivativos alternan, lo que confirma su identidad categorial: kiotogate-kiotazo, piñeragate-piñerazo, valijagate-maletinazo. 
- Dentro de este marco cabe, pues, diferenciar entre clases de unidades morfológicas (lexemas, morfemas gramaticales, morfemas derivativos, apreciativos, flexivos, elementos temáticos, morfos vacíos) y funciones morfológicas (RAíz, AFIJO y, como especificación de este último, PREFIJO, INFIJO, INTERFIJO, CIRCUNFIJO, SUFIJO).

- La utilización de esta doble perspectiva de análisis permite aportar soluciones en diversos aspectos del análisis morfológico (oposición lexema/raíz, los interfijos, falta de correspondencia biunívoca entre unidades y funciones morfológicas, transposición, etc.). Solo estudios empíricos posteriores podrán determinar el rendimiento real de este método de análisis.

\section{Referencias bibliográficas}

Aguirre, C. (2013). Manual de morfología del español. Barcelona: Castalia.

Aronoff, M. (1976). Word Formation in Generative Grammar. Boston: MIT Press.

Booij, G. E. (2005). The Grammar of Words: An Introduction to Linguistic Morphology. Oxford-New York: Oxford University Press.

Bosque, I. (1983). "La morfología”. En Abad, F. y A. García Berrio (eds.). Introducción a la lingüistica. Madrid: Alhambra, pp. 115-153.

Bosque, I. y V. Demonte (dirs.) (1999). Gramática descriptiva de la lengua española. Madrid: Espasa Calpe.

Bybee, J. L. (1985). Morphology: A Study of the Relation between Meaning and Form. Amsterdam: John Benjamins.

Coseriu, E. (1977). Principios de semántica estructural. Madrid: Gredos.

Coseriu, E. (1978). Gramátia, semántica, universales. Madrid: Gredos.

Fábregas, A. (2013). La morfología: el análisis de la palabra compleja. Madrid: Síntesis.

Fábregas, A. (2017). “¿Son algunos interfijos morfemas apreciativos?”, Estudios de Lingüística de la Universidad de Alicante, 31, pp. 135-150. DOI: 10.14198/ELUA2017.31.07

Felíu Arquiola, E. (2009). "Palabras con estructura interna". En De Miguel, E. (ed.). Panorama de la lexicología. Barcelona: Ariel, pp. 51-82.

Gutiérrez Ordóñez, S. (1991). La transposición sintáctica (problemas). Logroño: Gobierno de la Rioja.

Gutiérrez Ordóñez, S. (1997). Principios de sintaxis funcional. Madrid: Arco/Libros.

Hernanz, M. ${ }^{\mathrm{a}}$ Ll. y J. M. ${ }^{\mathrm{a}}$ Brucart (1987). La sintaxis 1: Principios teóricos. Barcelona: Crítica.

Lázaro Mora, F. A. (1999). "La derivación apreciativa”. En Bosque, I. y V. Demonte (dirs.), pp. 46654682.

Lyons, J. (1968). Introduction to Theoretical Linguistics. Cambridge University Press.

$N G L E=$ Real Academia Española y Asociación de Academias de la Lengua Española (2009). Nueva gramática de la lengua española. Morfología y sintaxis. Madrid: Espasa.

Otaola Olano, C. (2004). Lexicología y semántica léxica. Madrid: Ediciones Académicas.

Pena, J. (1999). "Partes de la morfología. Las unidades del análisis morfológico". En Bosque, I. y V. Demonte (dirs.), pp. 4305-4366.

Piera, C. y S. Varela (1999): "Relaciones entre morfología y sintaxis". En Bosque, I. y V. Demonte (dirs.), pp. 4367-4422.

Portolés, J. (1999). “La interfijación”. En Bosque, I. y V. Demonte (dirs.), pp. 5041-5073.

Rojo, G. y T. Jiménez Juliá (1989). Fundamentos del análisis sintáctico funcional. Santiago de Compostela: Servicio de publicaciones de la Universidad.

Scalise, S. (1984). Generative Morphology. Dordrecht: Foris.

Varela Ortega, S. (1990). Fundamentos de morfología. Madrid: Síntesis.

Varela Ortega, S. (2005). Morfología léxica: La formación de palabras. Madrid: Gredos. 
